\title{
Avaliação sensorial de sorvetes elaborados com leite de cabra
}

\section{Sensorial evaluation of ice-cream elaborated with goat milk}

\author{
Simone Cristina Pedrollo Lora ${ }^{1}$;Elane Schwinden Prudêncioº ${ }^{2}$ Honório Domingos Benedet $^{3 *}$
}

Resumo

O objetivo deste trabalho foi avaliar sensorial e economicamente formulações de sorvetes obtidos a partir do uso de leite de cabra em pó ou pasteurizado, gordura vegetal hidrogenada e aroma de morango. De vinte e sete formulações inicialmente previstas em um delineamento experimental, utilizando leite de cabra em pó, foram escolhidas as três melhores formulações. Estas três formulações foram avaliadas sensorialmente em três repetições utilizando leite de cabra em pó e três repetições utilizando o leite de cabra pasteurizado. Tanto para o leite em pó quanto para o pasteurizado as formulações escolhidas pelos julgadores foram as elaboradas com as seguintes variáveis: $80 \mathrm{~g}$ de gordura vegetal hidrogenada, 0,80 litros de leite de cabra (pó - reconstituído ou pasteurizado) e 0,12 g de aroma de morango. Estas formulações apresentaram Índice de aceitabilidade de 87,1 \% para o sorvete elaborado com leite de cabra em pó e 84,3\% para o leite de cabra pasteurizado. Foram realizadas as análises de custo, considerandose apenas os ingredientes da formulação com maior IA, que resultaram num custo de $\mathrm{R} \$ 3,15$ por litro e R\$ 2,68 para o sorvete de leite de cabra em pó e para o sorvete de leite de cabra pasteurizado, respectivamente.

Palavras-chave: Sorvete, leite de cabra, análise sensorial

\begin{abstract}
The objective of this work was to evaluate sensory and economically formulations obtained from of goat powder milk, vegetable fat and strawberry aroma. At least of twenty seven formulations listed in an experimental design, were choiced three best formulations, that they also were elaborated in triplicate to the powder goat milk and pasteurized goat milk. Finally it was choiced the best formulation. The final and the best ice-cream had the following formulation: $80 \mathrm{~g}$ of vegetable fat, $0.80 \mathrm{~L}$ of goat milk (powder reconstituted or pasteurized milk) and $0.12 \mathrm{~g}$ of strawberry aroma. These formulations shown Acceptability Index (AI) of $87.1 \%$ to ice-cream elaborated with powder milk and $84.3 \%$ to pasteurized milk. Considering just the last and the best AI formulation, it were realized the cost analysis of ice-creams. The price icecream with powder goat milk was equally to $\mathrm{R} \$ 3.15$ per liter, while with pasteurized milk was price per liter was equally to 2.68 per liter.
\end{abstract}

Key words: Ice-cream, goat milk, sensory analysis

1 Farmacêutica-Bioquímica, Mestre em Ciência dos Alimentos (CAL/CCA/UFSC).

2 Engenheira de Alimentos (UFSC), Doutora em Ciência de Alimentos (CAL/CCA/UFSC), Docente do Departamento de Ciência e Tecnologia de Alimentos do Centro de Ciências Agrárias da Universidade Federal de Santa Catarina.

3 Farmacêutico-Bioquímico (UFSC), Doutor em Ciência de alimentos (FEAA/UNICAMP), Docente voluntário do Departamento de Ciência e Tecnologia de Alimentos do Centro de Ciências Agrárias da Universidade Federal de Santa Catarina. Florianópolis/ SC.E-mail: benedet@cca.ufsc.br.

* Autor para correspondência.

Recebido para publicação 24/05/05 Aprovado em 10/10/05 


\section{Introdução}

O sorvete é uma mistura heterogênea, ao mesmo tempo emulsão, gel, suspensão e espuma, cuja coesão é mantida graças ao congelamento. Estruturalmente trata-se de uma espuma na qual as bolhas de ar estão cobertas por cristais de gelo, glóbulos de gordura individualizados ou parcialmente fundidos (grânulos gordurosos) e cristais de lactose. A estrutura dos glóbulos parcialmente fundidos e sua união às bolhas de ar dão ao sorvete firmeza residual depois da fusão dos cristais de gelo, sendo tal fato importante para os atributos sensoriais (PEREDA et al., 2005). Atualmente o sorvete é produzido em trocador de calor de superfície raspada, onde o ar é disperso através da mistura pelas lâminas do trocador, e mantidos sob congelamento (WILDEMOSER; SCHEIWILLER; WINDHAB, 2004). Desde sua invenção há séculos pelos chineses, vêm-se agregando ao sorvete novos sabores, textura, formas e processos tecnológicos de fabricação (GRANGER et al., 2005). Observa-se que fabricantes de sorvetes e de ingredientes caminham lado a lado em busca de novidades.

No Brasil, México, Argentina e Chile a caprinocultura leiteira além de não ser bem estabelecida (DUBEUF, 2005), compete com os produtos originários dos leites de vaca, ovelha e búfala (DUBEUF; MORAND-FEHR; RUBINO, 2004). Porém, o leite de cabra apresenta vantagens como glóbulos de gordura de menor tamanho (FRAZIER, 1995), hipoalergenicidade (ORTOLONI, 1997), alta digestibilidade (FRAZIER, 1995), um balanço de aminoácidos essenciais que eqüivale ou excede as recomendações da Organização Mundial de Saúde, com exceção do ácido fólico e vitamina $\mathrm{D}$, além de alto teor de cálcio, selênio, fosfato e vitaminas A e B (especialmente riboflavina) (ALFÉREZ et al., 2005). Outro aspecto de elevada importância na consideração do leite de cabra, em termos econômicos, é a alta conversão do leite em relação ao peso corporal das cabras, que podem resultar em uma produção leiteira durante dez dias equivalente ao seu peso (FURTADO, 1981).
O leite de cabra apresenta certas características próprias de qualidade que diferem do leite de outras origens, as quais fazem com que seja altamente recomendado na dieta infantil, de idosos e nos casos de alergia ao leite de vaca (ALFÉREZ et al., 2005). Raynal-Ljutovac, Gaborit e Lauret (2005) relatam que alguns critérios referentes à qualidade do leite $\mathrm{e}$ seus atributos sensoriais, como a lipólise, são levados em consideração na obtenção de derivados. A lipólise corresponde à hidrólise (enzimática) da matéria gorda do leite, que pode ser aumentada ou diminuída durante o processamento tecnológico do leite (RAYNALLJUTOVAC; ABORIT; LAURET, 2005). Tratamentos como a homogeneização e a armazenagem a frio, aumentariam a lipólise enquanto a pasteurização provocaria um decréscimo da mesma assim como o flavour desagradável creditado ao leite de cabra (MORGAN; GABORIT, 2001). Frazier (1995) afirmam também que o aquecimento do leite de cabra aumenta a sua digestibilidade e diminui ainda mais a sua hipoalergenicidade. Neves (1992) observou que o leite de cabra submetido ao tratamento térmico de desidratação (leite em pó), conseguiu manter praticamente inalterados os seus valores nutritivos e biológicos durante um período de 120 e 150 dias.

Ao contrário de países como a França, destaque na produção de queijo de cabra (MORAND-FEHR et al., 2003), a crescente demanda brasileira de leite de cabra é relacionada ao seu consumo na forma pasteurizada. Dubeuf (2005) afirma que derivados lácteos de cabra podem ser produzidos com qualidade e baixo custo desde que sejam incentivadas e pesquisadas novas alternativas para os mercados locais, nacionais e internacionais.

O objetivo deste trabalho foi avaliar sensorial e economicamente formulações de sorvetes obtidos a partir do uso de leite de cabra em pó, onde as melhores formulações foram reproduzidas também para sorvetes elaborados com leite de cabra pasteurizado. 


\section{Material e Métodos}

\section{Material}

Na fabricação dos sorvetes foi utilizado leite de cabra em pó integral (Scabra ${ }^{\circledR}$, CCA Laticínios Ltda, Rio de Janeiro - RJ, contendo em cada porção de 26g, 2 g de proteínas, 8 g de lipídios, 8 g de carboidratos, $235 \mathrm{mg}$ de cálcio e $70 \mathrm{mg}$ de sódio); e leite de cabra pasteurizado (Laticínios da Ama Ltda, Florianópolis - SC). Os leites foram gentilmente doados pelas empresas beneficiadoras. $\mathrm{Na}$ preparação da polpa de morango empregaram-se morangos sem agrotóxicos. Na elaboração dos sorvetes ainda foram utilizados gordura vegetal hidrogenada, açúcar, água e os seguintes aditivos, gentilmente cedidos pela Duas Rodas Industrial Ltda (Jaraguá do Sul - SC): Emustab ${ }^{\circledR}$, Liga neutra extra industrial $^{\circledR}$, Algemix $^{\circledR}$ e aroma de morango. Todos os reagentes utilizados foram de grau analítico P.A.

\section{Métodos}

Este trabalho foi dividido em duas etapas. A primeira etapa consistiu na elaboração de sorvetes com leite de cabra em pó, enquanto na segunda etapa foram comparadas as melhores formulações provenientes da primeira etapa, com sorvetes obtidos a partir do leite de cabra pasteurizado.

Análise físico-química do leite de cabra pasteurizado

Foram realizadas as seguintes análises físicoquímicas no leite de cabra pasteurizado: umidade (\% $\mathrm{p} / \mathrm{p})$, lipídios (\% p/p), proteínas (\% p/p), sais minerais $(\% \mathrm{p} / \mathrm{p})$, Extrato Seco Total (EST) $(\% \mathrm{p} / \mathrm{p})$, Extrato Seco Desengordurado (ESD) $(\% \mathrm{p} / \mathrm{p})$, acidez $\left({ }^{\circ} \mathrm{D}\right) \mathrm{e}$ densidade a $15{ }^{\circ} \mathrm{C}(\mathrm{g} / \mathrm{ml})$ (ASSOCIATION OF OFFICIAL ANALITYCAL CHEMISTS, 1998). Os teores de carboidratos $(\% \mathrm{p} / \mathrm{p})$ foram obtidos por diferença. As medidas do $\mathrm{pH}$ foram realizadas através do pH metro (MP220 Metler Toledo).

\section{Elaboração da calda de morango}

Para a elaboração da polpa de morango utilizouse $1 \mathrm{~kg}$ de morango para cada $100 \mathrm{~g}$ de sacarose. Esta mistura foi mantida em ebulição por 20 minutos, congeladas e armazenadas em câmara de congelamento a $-18{ }^{\circ} \mathrm{C}$.

Delineamento estatístico para o desenvolvimento das formulações de sorvetes de leite de cabra em pó

A fim de investigar o efeito de três variáveis independentes, teor de gordura vegetal hidrogenada, leite de cabra em pó (reconstituído de acordo com as instruções do fabricante) e aroma de morango, foram elaboradas vinte e sete formulações de sorvetes conforme o delineamento experimental apresentado na Tabela 1. Os demais ingredientes e aditivos foram mantidos constantes.

\section{Elaboração dos sorvetes}

Os sorvetes de leite de cabra em pó foram elaborados de acordo com as Técnicas Para Fabricação de Sorvetes e Picolés (DUAS RODAS INDUSTRIAL, 1998) (Figura 1), respeitando as variáveis constantes na Tabela 1. Os sorvetes foram acondicionados em baldes com capacidade para 2 litros, identificados por números de acordo com cada formulação (Tabela 1) e armazenados em câmara de congelamento à $25^{\circ} \mathrm{C}$ até a realização da análise sensorial.

Na segunda etapa o leite de cabra em pó foi substituído e empregado leite de cabra pasteurizado (Figura 1). Nesta etapa as três melhores formulações selecionadas pelos julgadores foram repetidas para o sorvete com leite de cabra em pó e leite de cabra pasteurizado. 
Tabela 1. Delineamento experimental para as formulações do sorvete de leite de cabra

\section{MATRIZ EXPERIMENTAL DOS SORVETES}

\begin{tabular}{|c|c|c|c|c|c|c|}
\hline \multirow[b]{2}{*}{ Experimento } & \multicolumn{3}{|c|}{ Variáveis Codificadas } & \multicolumn{3}{|c|}{ Variáveis Originais } \\
\hline & $\mathbf{X}_{1}$ & $\mathbf{X}_{2}$ & $\mathbf{X}_{3}$ & $\begin{array}{c}\text { Gordura vegetal } \\
\text { hidrogenada }(\mathrm{g})\end{array}$ & Leite de cabra ( $L)$ & $\begin{array}{c}\text { Aroma sabor } \\
\text { morango (g) }\end{array}$ \\
\hline 1 & -1 & -1 & -1 & 40 & 0,6 & 0,08 \\
\hline 2 & +1 & -1 & -1 & 80 & 0,6 & 0,08 \\
\hline 3 & -1 & +1 & -1 & 40 & 1,0 & 0,08 \\
\hline 4 & +1 & +1 & -1 & 80 & 1,0 & 0,08 \\
\hline 5 & 0 & 0 & -1 & 60 & 0,8 & 0,08 \\
\hline 6 & 0 & +1 & -1 & 60 & 1,0 & 0,08 \\
\hline 7 & +1 & 0 & -1 & 80 & 0,8 & 0,08 \\
\hline 8 & -1 & 0 & -1 & 40 & 0,8 & 0,08 \\
\hline 9 & 0 & -1 & -1 & 60 & 0,6 & 0,08 \\
\hline 10 & -1 & -1 & 0 & 40 & 0,6 & 0,10 \\
\hline 11 & +1 & -1 & 0 & 80 & 0,6 & 0,10 \\
\hline 12 & -1 & +1 & 0 & 40 & 1,0 & 0,10 \\
\hline 13 & +1 & +1 & 0 & 80 & 1,0 & 0,10 \\
\hline 14 & 0 & 0 & 0 & 60 & 0,8 & 0,10 \\
\hline 15 & 0 & +1 & 0 & 60 & 1,0 & 0,10 \\
\hline 16 & +1 & 0 & 0 & 80 & 0,8 & 0,10 \\
\hline 17 & -1 & 0 & 0 & 40 & 0,8 & 0,10 \\
\hline 18 & 0 & -1 & 0 & 60 & 0,6 & 0,10 \\
\hline 19 & -1 & -1 & +1 & 40 & 0,6 & 0,12 \\
\hline 20 & +1 & -1 & +1 & 80 & 0,6 & 0,12 \\
\hline 21 & -1 & +1 & +1 & 40 & 1,0 & 0,12 \\
\hline 22 & +1 & +1 & +1 & 80 & 1,0 & 0,12 \\
\hline 23 & 0 & 0 & +1 & 60 & 0,8 & 0,12 \\
\hline 24 & 0 & +1 & +1 & 60 & 1,0 & 0,12 \\
\hline 25 & +1 & 0 & +1 & 80 & 0,8 & 0,12 \\
\hline 26 & -1 & 0 & +1 & 40 & 0,8 & 0,12 \\
\hline 27 & 0 & -1 & +1 & 60 & 0,6 & 0,12 \\
\hline \multicolumn{4}{|c|}{ Variáveis Símbolos } & \multicolumn{3}{|c|}{ Níveis codificados } \\
\hline \multicolumn{4}{|c|}{ Independentes } & -1 & 0 & +1 \\
\hline \multicolumn{4}{|c|}{ Gordura vegetal hidrogenada $(\mathrm{g})$ : X1 } & 40 & 60 & 80 \\
\hline \multicolumn{4}{|c|}{ Leite de cabra (L): X2 } & 0,6 & 0,8 & 1,0 \\
\hline \multicolumn{4}{|c|}{ Aroma de morango $(\mathrm{g}): \mathrm{X} 3$} & 0,08 & 0,10 & 0,12 \\
\hline
\end{tabular}




\section{LEITE DE CABRA EM PÓ OU PASTEURIZADO}

(Adição de gordura, açúcar, água, Emustab ${ }^{\circledR}$, Liga neutra extra industrial ${ }^{\circledR}$, Algemix $^{\circledR}$ )

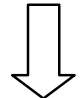

Agitação por 4 minutos

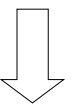

Resfriamento a $-10{ }^{\circ} \mathrm{C}$

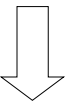

Batimento

(Adição de Algemix ${ }^{\circledR}$, calda de morango e aroma)

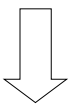

Maturação

(aprox. 30 minutos a $4{ }^{\circ} \mathrm{C}$ e leve agitação)

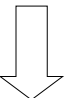

Batimento

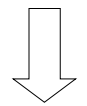

Envase

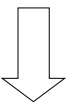

Armazenagem a $-25^{\circ} \mathrm{C}$

Figura 1. Fluxograma de elaboração dos sorvetes de leite de cabra. Fonte: Duas Rodas Industrial (1998).

\section{Análise sensorial}

As amostras de sorvete foram retiradas da câmara de congelamento $\left(-25^{\circ} \mathrm{C}\right)$ e deixadas por 30 minutos em congelador a $0{ }^{\circ} \mathrm{C}$, foram colocadas em forma de bolas em copos plásticos para serem avaliadas sensorialmente. Água foi servida aos julgadores para a limpeza do palato antes e entre as avaliações das formulações de sorvete.
$\mathrm{Na}$ primeira etapa as vinte e sete formulações foram divididas em três grupos com nove formulações cada, que foram identificadas por números, de $1 \mathrm{a}$ 27 (Tabela 1). A fim de evitar "fadiga" sensorial, o primeiro grupo (do número 1 ao 9), o segundo grupo (do número 10 ao 18) e o terceiro grupo (do número 19 ao 27), foram divididos também em três sessões (teste sensorial) composta por três formulações. De cada sessão obteve-se uma melhor formulação que 
ao final compreenderam as três melhores formulações de cada grupo. Estas formulações foram também avaliadas sensorialmente e resultaram em uma melhor formulação de cada grupo. Estas três últimas formulações finais foram então avaliadas sensorialmente em triplicata a fim de confirmar os resultados obtidos.

As três formulações selecionadas anteriormente, já na segunda etapa, foram repetidas, mas substituindo o leite de cabra em pó por leite de cabra pasteurizado, e então submetidas ao teste sensorial também em três repetições.

A aceitabilidade global das formulações de sorvete foi realizada por uma equipe de sete julgadores treinados, através do teste de escala hedônica de sete pontos, variando de $1=$ desgostei muito a $7=$ gostei muito (MEILGARD; CIVILLE; CARR, 1999).

Para as três formulações de sorvete de leite de cabra em pó e as três com leite de cabra pasteurizado foram calculados os Índices de Aceitabilidade, onde se considerou a pontuação máxima, nota 7 , como $100 \%$ e as médias atribuídas às melhores formulações o índice procurado.

\section{Análise estatística}

As médias, desvio padrão, análise de variância (ANOVA) (5\% de significância) e teste de Tukey foram calculadas através do software Statistica versão 6.0 (STATSOFT, 2001).

Análise de custo na elaboração do sorvete de leite de cabra

Para o cálculo da análise de custo dos sorvetes elaborados com leite de cabra em pó e leite de cabra pasteurizado, utilizou-se como parâmetro somente as formulações com maiores Índices de Aceitabilidade (IA). Esta análise foi realizada de acordo com Franco (1991) e não considerou levantamentos quanto à mãode-obra e os custos indiretos de fabricação.

\section{Resultados e Discussão}

Os resultados da análise físico-químico do leite de cabra pasteurizado utilizado nas formulações sorvetes da segunda etapa estão apresentados na Tabela 2. Estes resultados foram similares aos encontrados por Benedet e Carvalho (1996) diferindo somente no teor lipídico. Morand-Fehr (2005) afirma que a modificação na composição do leite de cabra é influenciada por fatores como raças, pelo regime alimentar adotado, clima, individualidade, entre outros.

Tabela 2. Resultado das médias (desvio padrão) referentes às características físicas e químicas do leite de cabra pasteurizado.

\begin{tabular}{lc}
\hline Características & Média (Desvio Padrão) \\
\hline Umidade $(\% \mathrm{p} / \mathrm{p})$ & $87,4(0,5)$ \\
Lipídios $(\% \mathrm{p} / \mathrm{p})$ & $4,2(0,1)$ \\
Proteínas $(\% \mathrm{p} / \mathrm{p})$ & $3,3(0,2)$ \\
Sais minerais $(\% \mathrm{p} / \mathrm{p})$ & $0,8(0,0)$ \\
Extrato Seco Total $(\mathrm{EST})(\% \mathrm{p} / \mathrm{p})$ & $12,6(0,5)$ \\
Extrato Seco Desengordurado $(\mathrm{ESD})(\% \mathrm{p} / \mathrm{p})$ & $8,3(0,4)$ \\
Acidez $\left({ }^{\circ} \mathrm{D}\right)$ & $18(1)$ \\
Densidade $\mathrm{a} 15^{\circ} \mathrm{C}(\mathrm{g} / \mathrm{mL})$ & $1,032(0,002)$ \\
Carboidratos totais $(\% \mathrm{p} / \mathrm{p})$ & $4,2(0,4)$ \\
$\mathrm{pH}$ & $6,6(0,1)$ \\
\hline
\end{tabular}


As médias das notas das vinte e sete formulações podem ser visualizadas na Tabela 3. As formulações escolhidas nas três sessões do primeiro grupo foram a 2, 4 e 8; do segundo grupo a 11, 13 e 16; e do terceiro grupo a 21, 22 e 25 . De cada grupo (total de três) foram retiradas às formulações com as melhores notas, ou seja, as formulações 2, 16 e 25. Estas formulações finais foram submetidas novamente aos testes sensoriais (em triplicata) a fim de se obter uma resposta considerada confiável para o sorvete de leite de cabra elaborado com leite de cabra em pó (Tabela 4). Segundo King (1994) e Frost et al. (2005) o sorvete é um alimento complexo de ser avaliado sensorialmente, sendo influenciado por vários fatores como o teor de gordura, aromas adicionados, o tipo de matéria-prima empregada, entre outros.

Tabela 3. Médias das notas dos julgadores da análise sensorial das vinte e sete formulações divididas em 9 grupos e estes em 3 sessões, respectivamente.

\begin{tabular}{cccc}
\hline Formulações & Grupos & Sessão & Médias das Notas dos Julgadores \\
\hline 1 & 1 & 1 & 3,6 \\
2 & 1 & 1 & $5,0^{* 1}$ \\
3 & 1 & 1 & 2,8 \\
4 & 1 & 2 & $4,8^{*}$ \\
5 & 1 & 2 & 4,7 \\
6 & 1 & 2 & 4,1 \\
7 & 1 & 3 & 4,8 \\
8 & 1 & 3 & $4,8^{*}$ \\
9 & 1 & 3 & 4,1 \\
10 & 2 & 1 & 3,4 \\
11 & 2 & 1 & $5,1 *$ \\
12 & 2 & 1 & 4,7 \\
13 & 2 & 2 & $4,7^{*}$ \\
14 & 2 & 2 & 4,4 \\
15 & 2 & 2 & 4,1 \\
16 & 2 & 3 & $5,4^{* 1}$ \\
17 & 2 & 3 & 4,7 \\
18 & 2 & 3 & 4,8 \\
19 & 3 & 1 & 4,3 \\
20 & 3 & 1 & 4,6 \\
21 & 3 & 1 & $5,0^{*}$ \\
22 & 3 & 2 & $5,3^{*}$ \\
23 & 3 & 2 & 5,1 \\
24 & 3 & 3 & 4,3 \\
25 & 3 & 3 & $6,3^{* 1}$ \\
26 & 3 & 3 & 4,7 \\
27 & 3 & & 5,0 \\
\hline
\end{tabular}

* Formulações escolhidas nas diferentes sessões.

${ }^{1}$ Formulações finais escolhidas em cada grupo. 
Em comum as formulações número 2, 16 e 25 apresentaram o maior teor de gordura vegetal hidrogenada adicionado. Este resultado é similar ao encontrado por Aime et al. (2001) cujos julgadores também escolheram formulações de sorvete com maiores teores de gordura. Bollinger et al. (2000), Aime et al. (2001) e Granger et al. (2005) afirmam que o maior teor de gordura confere cremosidade, proporciona textura suave e encorpa o sorvete, influenciando, portanto na escolha do produto. Já, as quantidades de leite de cabra em pó e aroma escolhidas foram variáveis.

Das três melhores formulações pode-se verificar também na Tabela 4 que a formulação escolhida pelos julgadores foi a de número 25 , onde se utilizou os maiores teores de gordura vegetal hidrogenada ( 80 g) e aroma ( $0,12 \mathrm{~g})$, e valores intermediários de leite de cabra $(0,80 \mathrm{~L})$. O mesmo comportamento foi verificado com os sorvetes elaborados com leite de cabra pasteurizado (Tabela 4). Koeferli, Piccinali e Sigrist (1996) relatam que o off flavour de certos leites e produtos lácteos, como o sorvete, armazenados em baixas temperaturas, é melhorado também através da adição de gordura. A melhora da aceitabilidade de sorvetes vem sendo incentivada também através da adição de aromas que de acordo com Pereda et al. (2005) contribuem para realçar o sabor.

Tabela 4. Médias das notas das três melhores formulações de sorvete elaboradas com leite de cabra em pó reconstituído e leite de cabra pasteurizado, realizadas em triplicata.

\begin{tabular}{ccc}
\hline Formulação & \multicolumn{3}{c}{ Médias das Notas dos Julgadores } \\
\hline & Sorvete com leite de cabr em pó reconstituído & Sorvete de leite de cabra pasteurizado \\
\hline 2 & $4,5^{\mathrm{a}}$ & $4,6^{\mathrm{a}}$ \\
16 & $4,8^{\mathrm{a}}$ & $4,9^{\mathrm{a}}$ \\
25 & $6,1^{\mathrm{b}}$ & $5,9^{\mathrm{b}}$ \\
\hline
\end{tabular}

* Médias com a mesmas letras nas mesmas colunas não diferem significativamente ( $\mathrm{p}>0,05)$.

Os valores obtidos para o Índice de Aceitabilidade (IA) das três melhores formulações (Tabela 5) indicam que tanto para os sorvetes elaborados com leite de cabra em pó como o pasteurizado a formulação número 25 foi a que apresentou maior aceitabilidade pelos julgadores. A formulação número 16 somente foi aceita para o leite de cabra pasteurizado, enquanto a formulação número 2 não foi aceita pelos julgadores. A não aceitação da formulação número 16 utilizando leite de cabra em pó poderia estar ligada à elevada temperatura empregada na desidratação do leite. Segundo Pereda et al. (2005) na obtenção de leite em pó, observa-se uma maior reação de Maillard do que no processo de pasteurização.

Tabela 5. Índice de Aceitabilidade (IA) dos sorvetes de leite de cabra em pó reconstituído e pasteurizado das melhores formulações.

\begin{tabular}{ccc}
\hline Formulação & \multicolumn{3}{c}{ Índice de Aceitabilidade (\%) } \\
\hline & Sorvete com leite de cabra em pó reconstituído & Sorvete de leite de cabra pasteurizado \\
\hline 2 & 64,3 & 65,7 \\
16 & 68,6 & 70,0 \\
25 & 87,1 & 84,3 \\
\hline
\end{tabular}


Para a análise de custos dos sorvetes de leite de cabra em pó e pasteurizado utilizou-se à formulação com maior IA, ou seja, a formulação número 25 . A Tabela 6 mostra a relação, a quantidade e o custo das matérias-primas, ingredientes e aditivos utilizados na obtenção de 1,5 litros de sorvete de leite de cabra em pó e pasteurizado. Os preços para cada litro de sorvete seriam de $\mathrm{R} \$ 3,15$ e $\mathrm{R} \$ 2,68$, para o elaborado com leite de cabra em pó e pasteurizado, respectivamente.

Tabela 6. Relação de componentes, quantidade, valores e custos para a produção de 1,5 litros de sorvete de leite de cabra em pó e pasteurizado.

\begin{tabular}{|c|c|c|c|c|}
\hline & Quantidades & Unidade & $\begin{array}{c}\text { Valor Unitário } \\
(\mathbf{R} \$)\end{array}$ & Valor Total (R\$) \\
\hline Emustab $^{\circledR}$ & 10 & $\mathrm{~g}$ & 0,0114 & 0,1140 \\
\hline $\begin{array}{l}\text { Liga neutra extra } \\
\text { industrial }^{\circledR}\end{array}$ & 3 & $\mathrm{~g}$ & 0,0128 & 0,0384 \\
\hline $\begin{array}{l}\text { Gordura vegetal } \\
\text { hidrogenada }\end{array}$ & 80 & $\mathrm{~g}$ & 0,0032 & 0,2560 \\
\hline Algemix $^{\circledR}$ & 24 & $\mathrm{~g}$ & 0,0078 & 0,1872 \\
\hline Aroma de morango & 12 & $\mathrm{~g}$ & 0,0072 & 0,0864 \\
\hline Calda de morango & 100 & $\mathrm{~g}$ & 0,00579 & 0,5790 \\
\hline Açúcar cristal & 200 & $\mathrm{~g}$ & 0,0013 & 0,2600 \\
\hline $\begin{array}{l}\text { Leite de cabra em pó } \\
\text { (a ser reconstituído) }\end{array}$ & 80 & $\mathrm{~g}$ & 0,0400 & 3,2000 \\
\hline $\begin{array}{l}\text { Leite de cabra } \\
\text { pasteurizado }\end{array}$ & 1 & $\mathrm{~L}$ & 2,5000 & 2,5000 \\
\hline $\begin{array}{l}\text { *Sorvete de leite de } \\
\text { cabra em pó }\end{array}$ & 1,5 & $\mathbf{L}$ & - & 4,7200 \\
\hline $\begin{array}{l}* \text { Sorvete de leite de } \\
\text { cabra pasteurizado }\end{array}$ & 1,5 & $\mathbf{L}$ & - & 4,0210 \\
\hline
\end{tabular}

* Não foram levados em consideração levantamentos quanto à mão-de-obra e os custos indiretos de fabricação.

\section{Conclusão}

Através dos resultados obtidos deste trabalho pode-se concluir que é possível elaborar sorvetes de leite de cabra em pó e pasteurizado sensorialmente aceitos. A melhor formulação de sorvete elaborado com leite de cabra em pó (reconstituído) e pasteurizado foi à mesma, ou seja, a que se utilizou as seguintes variáveis testadas: $80 \mathrm{~g}$ de gordura vegetal hidrogenada, 0,80 litros de leite de cabra (pó - reconstituído ou pasteurizado) e 0,12 g de aroma de morango (formulação número 25).

Os maiores Índices de Aceitabilidade (IA) encontrados foram para a formulação número 25 , sendo iguais a $87,1 \%$ para o sorvete elaborado com leite de cabra em pó e 84,3\% para o leite de cabra pasteurizado.
As análises de custo, considerando-se apenas os ingredientes da formulação com maior IA foram de $\mathrm{R} \$ 3,15$ por litro para o sorvete de leite de cabra em pó e $\mathrm{R} \$ 2,68$ por litro para o sorvete de leite de cabra pasteurizado.

\section{Agradecimentos}

Agradecemos a CCA Laticínios Ltda. (Rio de Janeiro - RJ), a Laticínios da Ama Ltda. (Florianópolis - SC), a Duas Rodas Industrial (Jaraguá do Sul - SC), a CAPES e a UFSC (CCA/CAL/ PGCAL). 


\section{Referências}

AIME, D. B.; ARNTFIELD, S. D.; MALCOMSON, L. J.; RYLAND, D. Textural analysis of fat reduced vanilla ice cream products. Food Research International, Barking, Inglaterra, v.34, n.2/3, p.237-246, 2001.

ALFÉREZ, M. J. M.; LÓPEZ-ALIAGA, I.; NESTARES, T.; DIAZ-CASTRO, J.; BARRIONUEVO, M.; ROSS, P. B.; CAMPOS, M. S. Dietary goat milk improves iron bioavailability in rats with induced ferropenic anaemia incomparison with cow milk. Journal of Dairy Research, Cambridge, v.70, p.181-1872003.

ASSOCIATION OF OFFICIAL ANALITYCAL CHEMISTS. Official methods of analysis. $14 \mathrm{ed}$. Washington: AOAC, 1998.

BENEDET, H. D.; CARVALHO, M. W. Caracterização do leite de cabra no estado de Santa Catarina, Brasil. Ciência e Tecnologia de Alimentos, Campinas, v.16, p.100-107, 1996.

BOLLIGER, S.; KORNBRUST, B., GOFF, H. D.; THARP, B. W.; WINDHAB, E. J. Influence of emulsifiers on ice cream produced by conventional freezing and low temperature extrusion processing. International Dairy Journal, Barking, Inglaterra, v.10, n.7, p.497-504, 2000.

DUAS RODAS INDUSTRIAL. Técnicas para fabricação de sorvetes e picolés. Jaraguá do Sul: DUAS RODAS, 1998.

DUBEUF, J. P. Structural, market and organizational conditions for developing goat dairy production systems. Small Ruminant Research, Amsterdam, v.60, p.67-74, 2005.

DUBEUF, J. P.; MORAND-FEHR, P.; RUBINO, R. Situation, changes and future of goat industry around the world. Small Ruminant Research, Amsterdam, v.51, n.2, p.165173, 2004.

FRANCO, H. Contabilidade Industrial. São Paulo: Atlas, 1991.

FRAZIER, C. A. Food allergies got your goat? A "nanny" may help wean grown-ups from milk. Total Health, v.17, p.46-47, 1995.

FROST, M. B.; HEYMANN, H.; BREDIE, W. L. P.; DIJKSTERHUIS, G. B.; MARTENS, M. Sensory measurement of dynamic flavour intensity in ice cream with different fat levels and flavourings. Food Quality and Preference, Barking, Inglaterra, v.16, n.4, p.305-314, 2005.

FURTADO, M. M. Leite de cabra: características especiais. Seu uso na alimentação. Intolerância. Revista do Instituto de Laticínios Cândido Tostes, Juiz De Fora, v.214, p.3133, 1981.
GRANGER, C.; LEGER, A.; BAREY, P.; LANGENDORFF, $\mathrm{V}$.; CANSELL, M. Influence of formulation on the structural networks in ice cream. International Dairy Journal, Barking, Inglaterra, v.15, n.3, p.255-262, 2005.

KING, B. M. Sensory profiling of vanilla ice cream: flavour and base interactions. Lebensmittel-Wissenschaft undTechnologie, London, v.27, p.450-456, 1994.

KOEFERLI, C. R. S.; PICCINALI, P.; SIGRIST, S. The influence of fat, sugar and non-fat milk solids on selected taste, flavor and texture parameters of a vanilla ice-cream. Food Quality and Preference, Barking, v.7, n.2, p.69-79, 1996.

MEILGAARD, M.; CIVILLE, G. V.; CARR, B. T. Sensory evaluation techniques. 3. ed. Boca Raton: FL:CRC, 1999.

MORAND-FEHR, P. Recent developments in goat nutrition and application: a review. Small Ruminant Research, Amsterdam, v.60, n.1/2, p.25-43, 2005.

MORAND-FEHR, P.; BOUTONNET, J. P.; DEVENDRA, C.; DUBEUF, J. P.; HAEN-LEIN, G.; HOLST, P.; LAMBOLEY-GA“ UZERE, B.; MOWLEM, L.; CAPOTE, J. Introduction for strategy for development of goat farming in the 21st century. Small Ruminant Research, Amsterdam, v.51, n.2, p.175-183, 2003.

MORGAN, F.; GABORIT, P. The typical flavour of goat milk products: technological aspects. International Journal of Dairy Technology, Huntingdon, Inglaterra, v.54, n.1, p.38-40, 2001.

NEVES, R. Leite em pó valoriza a caprinocultura. Manchete Rural, Rio De Janeiro, v.5, n.64, p.42-45, 1992.

ORTOLONI, E. L. Porque adultos não bebem leite. Balde Branco, Sao Paulo, v.32, n.391, 1997.

PEREDA, J. A. O.; RODRÍGUEZ, M. I. C.; ÁLVAREZ, L. F. ; SANZ, M. L. G.; MINGUILLÓN, G. D. G. F.; PERALES, L. H.; CORTECERO, M. D. S. Tecnologia de alimentos, São Paulo: Artmed, 2005. v.2

RAYNAL-LJUTOVAC, K.; GABORIT, P.; LAURET, A. The relationship between quality criteria of goat milk, its technological properties and the quality of the final products. Small Ruminant Research, Amsterdam, v.60, n.1/2, p.167-177, 2005.

STATSOFT. STATISTICA (data analysis system) versão 6.0. Tulsa: STATSOFT Inc, 2001.

WILDEMOSER, H.; SCHEIWILLER, J.; WINDHAB, E. J. Impact of disperse microstructure on rheology and quality aspects of ice cream. Lebensmittel-Wissenschaft undTechnologie, London, v.37, p.881-891, 2004. 